\title{
Proteus Syndrome: About Two Paediatrics Cases
}

\author{
Senkaye-Lagom Aimée Kissou', Aïda Tankoano², Ollo Roland Somé3, \\ Amina Nomtondo Ouédraogo ${ }^{4}$, Patrick Wendpouiré Hamed Dakouré3, \\ Zakary Nikiema ${ }^{2}$, Boubacar Nacro ${ }^{1}$
}

\footnotetext{
${ }^{1}$ Department of Pediatrics, Sourô Sanou University Teaching Hospital (CHUSS), Bobo-Dioulasso, Burkina Faso

${ }^{2}$ Medical Imaging Department, Sourô Sanou University Teaching Hospital (CHUSS), Bobo-Dioulasso, Burkina Faso

${ }^{3}$ Department of Surgery, Sourô Sanou University Teaching Hospital (CHUSS), Bobo-Dioulasso, Burkina Faso

${ }^{4}$ Department of Dermatology, Yalgado Ouédraogo University Teaching Hospital, Ouagadougou, Burkina Faso

Email: aimekissou@yahoo.fr
}

How to cite this paper: Kissou, S.-L.A., Tankoano, A., Somé, O.R., Ouédraogo, A.N., Dakouré, P.W.H., Nikiema, Z. and Nacro, B. (2018) Proteus Syndrome: About Two Paediatrics Cases. Open Journal ot Pediatrics, 8, 42-49.

https://doi.org/10.4236/ojped.2018.81006

Received: December 25, 2017

Accepted: March 5, 2018

Published: March 8, 2018

Copyright (c) 2018 by authors and Scientific Research Publishing Inc. This work is licensed under the Creative Commons Attribution International License (CC BY 4.0).

http://creativecommons.org/licenses/by/4.0/

\begin{abstract}
Proteus syndrome (PS) is a rare pathology characterized by mosaic and progressive hypertrophies, that can affect any organ or tissue of the body. A genetic mutation in the embryo is at the origin of the disease. Bones, fat, skin and connective tissue are the tissues most typically involved. The extent and severity of the abnormalities vary, giving different clinical presentations. The authors report two cases in two boys aged three and eight years respectively. In both, segmental and organ growth abnormalities, skin abnormalities and lipomas were present. The youngest patient was admitted for the management of severe malnutrition. He presented a major excrescence in the gluteal region, which required surgical management mainly because of its functional impact. In the second patient who was hospitalized following a viral meningoencephalitis, important vascular abnormalities were demonstrated, particularly at the cerebral and abdominal level. The clinical presentation was more severe in the latter, with the installation of an extended coma and a comitial state. PS is a pathology which could be incapacitating. It exposes to a high risk of benign tumours and deep venous thrombosis. The variability of abnormalities and the risks incurred required multidisciplinary management.
\end{abstract}

\section{Keywords}

Proteus, Growth Abnormalities, Cutaneous Signs, Vascular Abnormalities

\section{Introduction}

Proteus syndrome (PS) is a rare pathology characterised by a mosaic progressive 
hypertrophy which could affect any organ or any tissue of the body. An embryonic genetic mutation could be the causes. Bones, fat, skin, and conjunctive tissue are more typically involved. Extent and severity of the disease vary from one individual to another, giving different clinical presentations. The authors report two cases of two boys aged respectively 3 and 8 years.

\subsection{Observation 1}

A three years old boy, the last of 6 children, without any known family history of congenital disease, was admitted with severe malnutrition (z-score $\mathrm{P} / \mathrm{T}<-3 \mathrm{ET}$ ) complicated by anemia. The child was cachectic with tiny limbs and macrocephaly. The examination shows a right side body hemi-hypertrophy with asymmetrical ears (Figure 1(a)) and feet.

The plantar surface of the right foot was cribriform. An epidermal naevus was seen at the right cervical region and homolateral ear (Figure 1(b)).

There was an extra growth at the gluteal region and the right thigh affecting walking and some postures like sitting (Figure 2(a) \& Figure 2(b)).

CT scan of the growth shows a huge heterogeneous subcutaneous soft tissue mass poorly circumscribed measuring $196 \times 55 \mathrm{~mm}$, with an important fatty tissue poorly enhanced by injected contrast, attached to the gluteal muscles. There was right ileo-femoral dysplasia with ascending of the femoral head. Abdomino-pelvic CT scan show a big right kidney, sub-renal lymphangioma and right hydrocele. Other intra-abdominal organs and vessels were normal; there was neither intra-abdominal mass nor ascites. The association of asymmetry growth, epidemics naevus, lipoma, macrocephaly and bony abnormalities contributed in making the diagnosis of PS.

After management of acute complications and prolong dietary treatment, there was an improvement of general and nutritional status of the patient. After one year, a surgical excision of the gluteal and thigh mass was done (Figure 3).
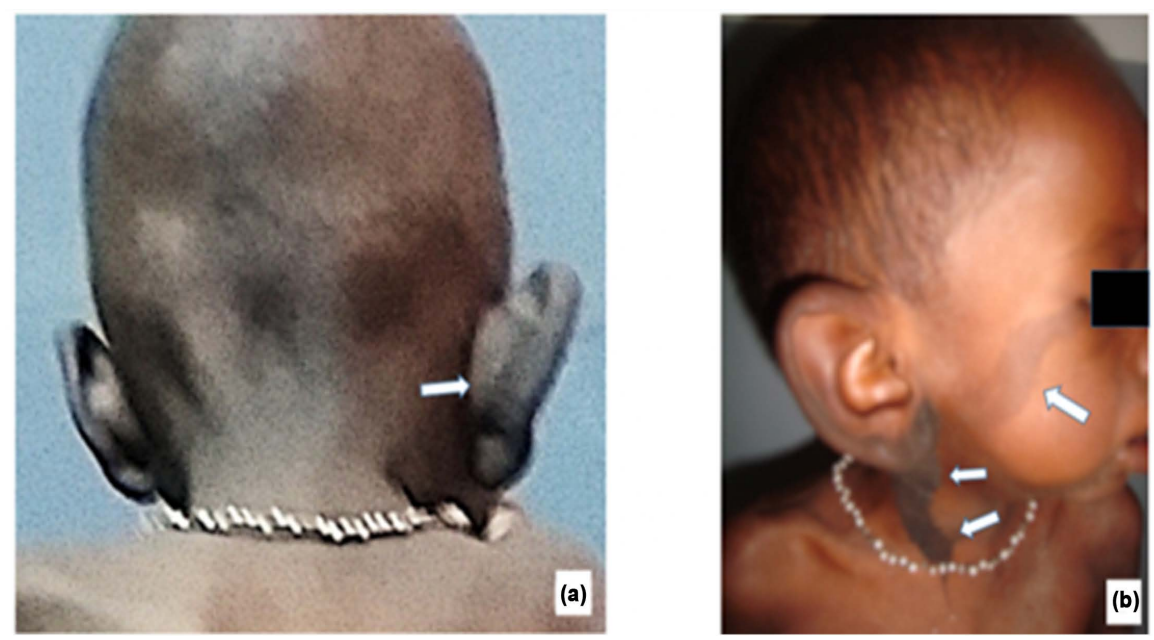

Figure 1. Patient's head photographs back (a) and profile (b) showing a right hypertrophied ear (a): arrow) associated to an epidermal naevus on the right cervical region and homolateral ear (b): arrows). 

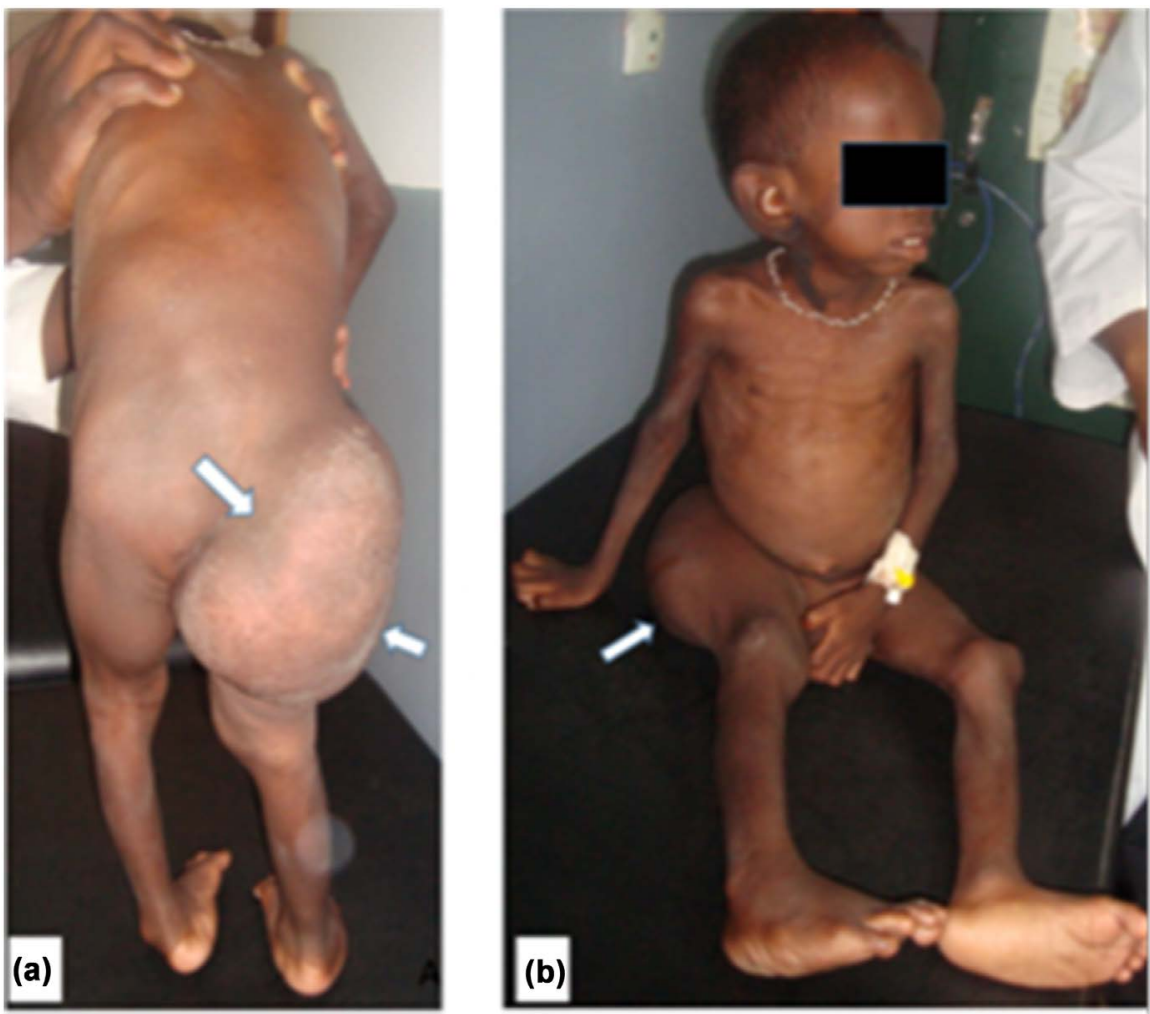

Figure 2. Patients' photographs back (a) and face (b) showing the masse in the right gluteal region (arrows).
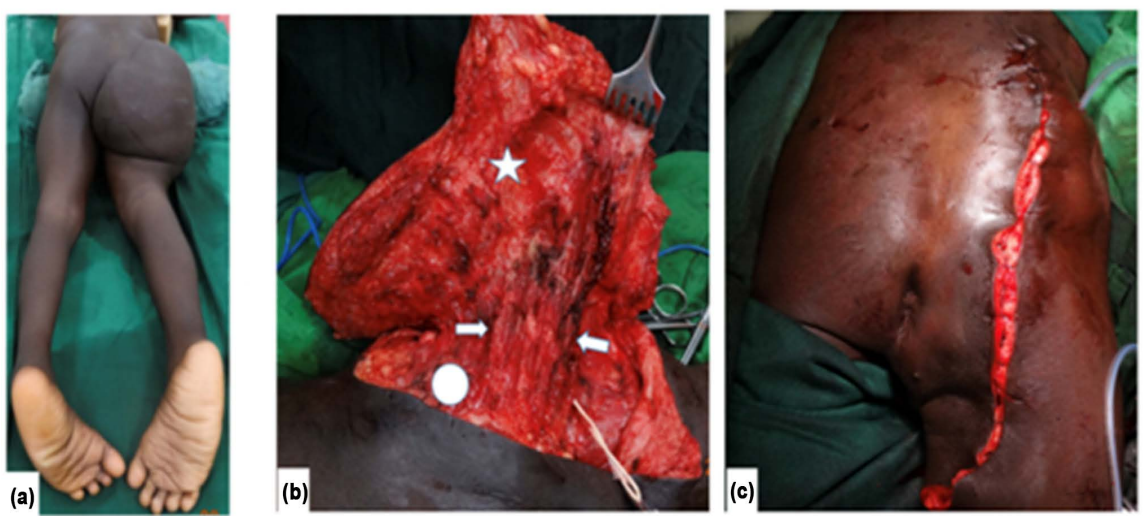

Figure 3. photographs showing the surgical procedure; patient position (a), excision of the mass (star) showing attachment (Arrows) of the mass to the major gluteal muscle (circle) and covering of the site of the exeresis (c).

The result was satisfactory with esthetical and functional improvement (Figure 4).

\subsection{Observation 2}

An eight years old boy, only child from the mother, was admitted with history of coma and febrile convulsions. At admission, examination found frank meningeal syndrome. Cerebro-spinal fluid analysis showed high protein level and 

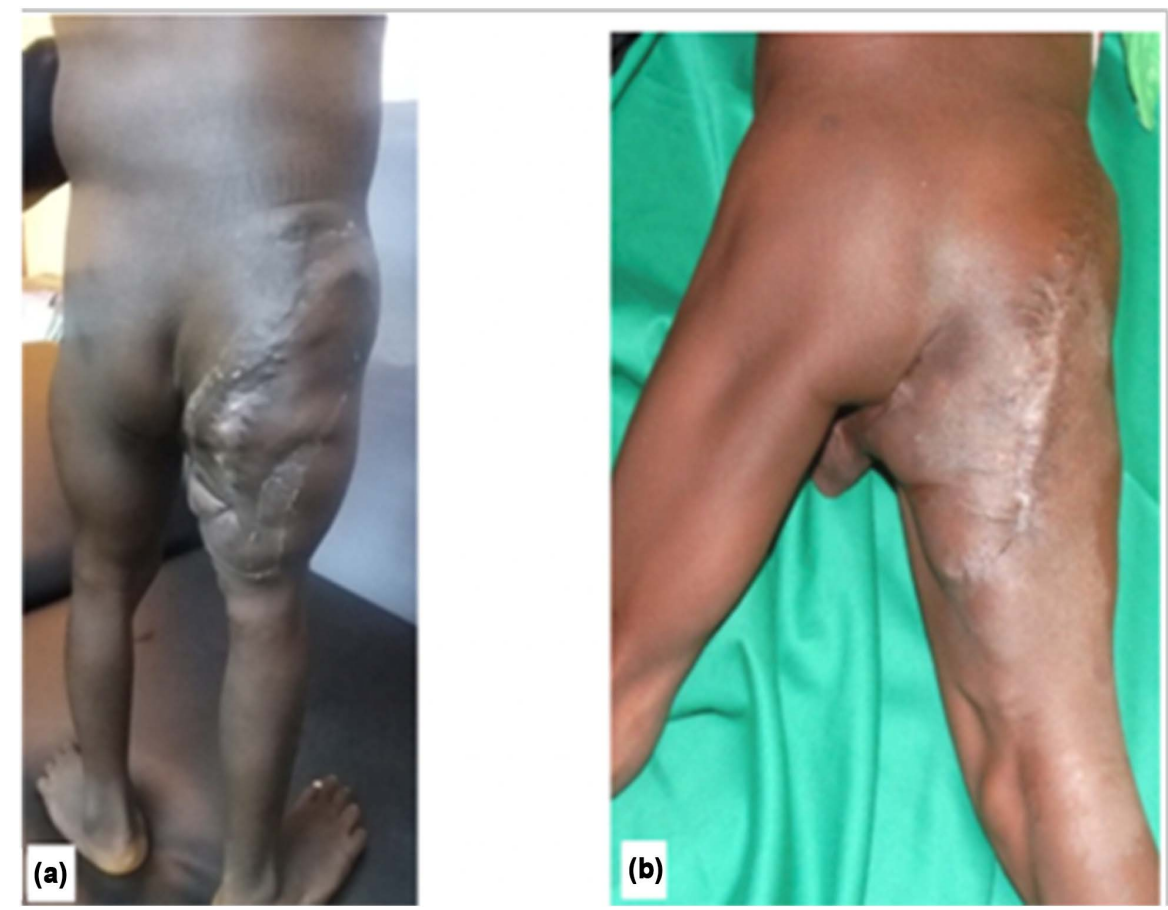

Figure 4. Functional and esthetic results.

discrete leucocytosis with mostly lymphocytosis. The diagnosis of viral meningo-encephalitis was retained.

Blood culture was negative. Few days after hospitalization, the patient developed varicella. Examination shows numerous morphological abnormalities: right hemi thorax soft tissue overgrowth, with a verruca naevus adjacent but extending down to the abdomen; there was coexisting hypertrophy of the right buttocks and the right foot (Figure 5).

Cerebral CT scan (Figure 6) showed:

$\checkmark$ an important compressive bi-cerebral hygroma of $13 \mathrm{~mm}$ thickness;

$\checkmark$ bilateral capsular lacuna;

$\checkmark$ third ventricular hydrocephaly;

$\checkmark$ multifocal arterio-veinous malformations.

The concomitant presence of naevus, asymmetrical growth, lipoma and vascular abnormalities called for PS.

Evolution was stagnant with no recovery of the level of consciousness with persistent convulsive crisis despite permanent anti-convulsive therapy. There was improvement in temperature. Patient was discharged against medical advice on relatives' request.

\section{Discussion}

PS is a rare disease of unknown aetiology. It is part of malformative syndrome with excessive growth and frequently associated with genetic anomalies [1]. PTEN (tumour suppressive gene) mutation have been found in some patients, but not confirmed in majority of examined cases [2]. While a somatic gene 

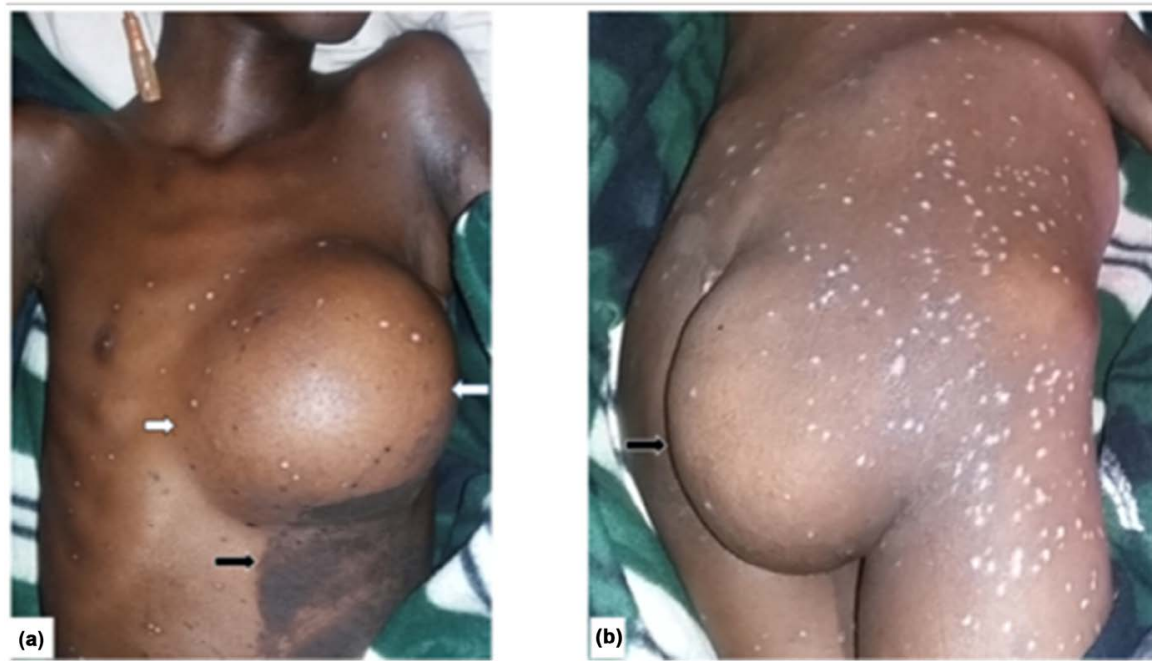

Figure 5. Photographs of the patient trunk showing a mass (white arrows on Figure 5(a)) and verruca naevus adjacent but extending dawn to the abdomen (black arrows).

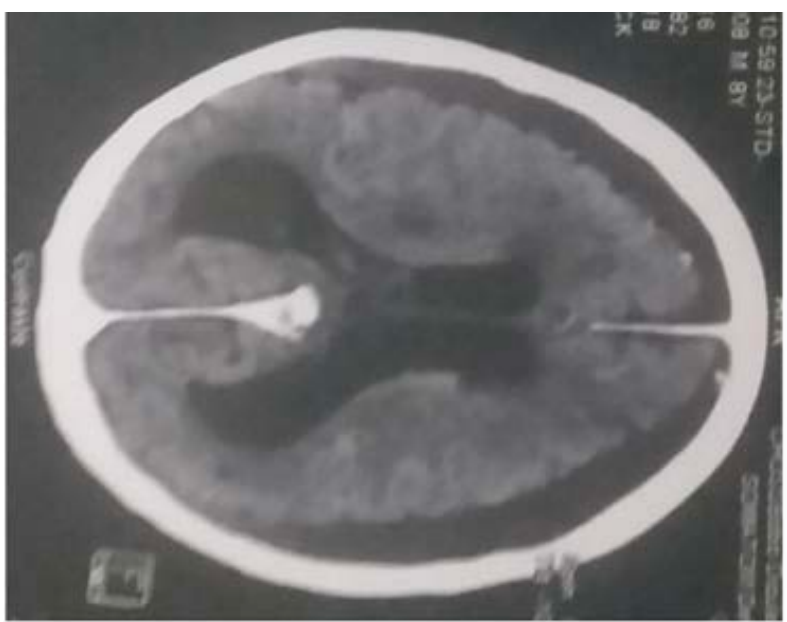

Figure 6. Cerebral CT scan of patient n 2 .

mutation AKTI was in all patients with PS [3]. This gene is oncogenic and codes for AKT1 kinase, an important enzyme in the regulation of cellular proliferation and apoptosis, explaining overgrowth and propensity to develop tumours. Growth anomalies are diagnostic criteria of PS. However there is a controversy on the definition.

A review of 250 cases was separately published by some authors who decided to apply the already existing criteria to those different cases. Only $47.3 \%$ of patients were considered reel cases of PS [4]. This review permits the development of the diagnostic criteria established in 1998 by American institute of health [5]. Several propositions exist, but globally the diagnosis is based on general and specific criteria [4] [6] [7]. General criteria are mosaic distribution of lesions, their progressive evolution and sporadic appearance of some cases (not inherited). Specific criteria are classified into 3 categories (A, B and C). Those criteria are from growth anomalies to conjunctive tissue and skin involvement, specific 
tumours and vascular malformations, lipoma and others dysmophysms. Table 1 which is a summary of diagnostic criteria of PS is a simplified version of which published by Biesecker in 2006 [7].

To make a diagnosis of PS, one must have all the general criteria, and various specific criteria

${ }^{*}$ Adapted from Biesecker publication [7]

Growth anomalies, like others signs are generally absent at birth. They appear and increased progressively and could affect all part of the body; limbs, sensory organs, (ears in our first patient), deep organs could be asymmetric. Involvement of visible part could be disgracing.

Lipomatous overgrowths are frequent and could simulate a royal tumour or carcinomatous degeneration in these polymorphic syndromes [8]. Moreover, some disturbing characters and localisation call for surgical intervention like in our two cases that had the growth at the gluteal region making difficulty in sitting and walking. This justified the surgical intervention for the first patient. Excision biopsy in block for diagnostic and therapeutic and oncologic management was rapidly confronted with difficulties. In fact, a diffused infiltration of fibrous tumour tissue was noted into and under skin, and penetrating sometimes muscles, as shown the CT SCAN. Benign aspect was suggested while waiting for confirmation from the histology which later confirms a benign fibrous mesenchymal tissue. Vascular malformations of PS involved veins than artery. Our second patient had complex cerebral and abdominal arterio-veinous malformations.

Clinical presentation varied extremely in different cases published involving both children and adults [9] [10].

Differential diagnostic could be made with others syndromes with the triads: growth anomalies, cutaneous signs, and vascular malformations. Could be cited: type 1 neurofibromatosis (NF1), body hemi-hypertrophy and multiple lipomatous syndrome (HHML) [7].

The fact that PS is part of a big group of pathology caused by somatic mosaic mutation and not hereditary must lead to be careful in making the diagnosis. In fact, the relationship between somatic mutation and carcinoma has been largely

Table 1. Synthesis of diagnosis criteria of Proteus Syndrome.

\begin{tabular}{ll}
\hline General criteria & $\begin{array}{l}\text { Mosaic distribution of lesions } \\
\text { Sporadic occurrence } \\
\text { Progressive course }\end{array}$ \\
& A: 1) Cerebriform connective tissue nevus \\
B: 1) Linear epidermal nevus \\
2) Asymmetric, disproportionate overgrowth \\
3) Specific tumors before 2nd decade (Bilateral ovarian \\
cystadenoma; Parotid monomorphic adenoma) \\
C: 1) Dysregulated adipose tissue \\
2) Vascular malformations \\
3) Lung cysts \\
4) Facial phenotype
\end{tabular}


studied and lists of complete oncogenes or tumour suppressor genes known to be mutated in cancers has been described [11].

PS complications depend on the existing anomalies, but it is known that the major risks are deep venous thrombosis and tumours. Pulmonary embolism is a frequent complication in patients, implicating the necessity of careful precautions, especially during surgery. The two principal tumours known to be part of specific diagnostic criteria are bilateral ovarian cyst adenoma and parotids glands monomorphic adenoma. A recent publication suggested some immune disturbances related to PS [12]. That is maturation and activation anomalies of T and B lymphocytes. From there, vulnerable infections could arise. The variability of clinical presentations and multi-organs involvement as well as the risks on the patients with PS required multidisciplinary management.

\section{Conclusion}

PS is a pathology which could be incapacitating. It exposes to a high risk of benign tumours and deep venous thrombosis. The variability of abnormalities and the risks incurred required multidisciplinary management.

\section{References}

[1] Edmondson, A.C. and Kalish J.M. (2015) Overgrowth Syndromes. Journal of Pediatric Genetics, 4, 136-143. https://doi.org/10.1055/s-0035-1564440

[2] Zhou, X., Hampel, H., Thiele, H., Gorlin, R.J., Hennekam, R.C., Parisi, M., Winter, R.M. and Eng, C. (2001) Association of Germline Mutation in the PTEN Tumour Suppressor Gene and Proteus and Proteus-Like Syndromes. Lancet, 358, 210-211. https://doi.org/10.1016/S0140-6736(01)05412-5

[3] Lindhurst, M.J., Sapp, J.C., Teer, J.K., Johnston, J.J., Finn, E.M., Peters, K., Turner, J., Cannons, J.L., Bick, D., et al. (2011) A Mosaic Activating Mutation in AKT1 Associated with the Proteus Syndrome. The New England Journal of Medicine, 365, 611-619. https://doi.org/10.1056/NEJMoa1104017

[4] Turner, J.T., Cohen Jr, M.M. and Biesecker, L.G. (2004) Reassessment of the Proteus Syndrome Literature: Application of Diagnostic Criteria to Published Cases. American Journal of Medical Genetics, Part A, 130A, 111-122. https://doi.org/10.1002/ajmg.a.30327

[5] Biesecker, L.G., Happle, R., Mulliken, J.B., Weksberg, R., Graham Jr, J.M., Viljoen, D.L. and Cohen Jr, M.M. (1999) Proteus Syndrome: Diagnostic Criteria, Differential Diagnosis, and Patient Evaluation. American Journal of Medical Genetics, Part A, 84, 389-395. https://doi.org/10.1002/(SICI)1096-8628(19990611)84:5<389::AID-AJMG1>3.0.CO; $\underline{2-\mathrm{O}}$

[6] Cohen Jr, M.M. (2005) Proteus Syndrome: An Update. American Journal of Medical Genetics. Part C, Seminars in Medical Genetics, 137C, 38-52. https://doi.org/10.1002/ajmg.c.30063

[7] Biesecker, L. (2006) The Challenges of Proteus Syndrome: Diagnosis and Management. European Journal of Human Genetics, 14, 1151-1157. https://doi.org/10.1038/sj.ejhg.5201638

[8] Erickson, R.P. (2014) Recent Advances in the Study of Somatic Mosaicism and Dis- 
eases Other than Cancer. Current Opinion in Genetics \& Development, 26, 73-78. https://doi.org/10.1016/j.gde.2014.06.001

[9] Lal, N.R., Bandyopadhyay, D. and Sarkar A.K. (2015) Unilateral Hypertrophic Skin Lesions, Hemimegalencephaly, and Meningioma: The Many Faces of Proteus Syndrome. Indian Dermatology Online Journal, 6, 348-351. https://doi.org/10.4103/2229-5178.164477

[10] Ou, M.L., Sun, Z.J., Zhu, P., Sun, G.P. and Dai, Y. (2017) Proteus Syndrome: A Case Report and Review of the Literature. Molecular and Clinical Oncology, 6, 381-383. https://doi.org/10.3892/mco.2017.1140

[11] Freed, D., Stevens, E.L. and Pevsner, J. (2014) Somatic Mosaicism in the Human Genome. Genes, 5, 1064-1094. https://doi.org/10.3390/genes5041064

[12] Lougaris, V., Salpietro, V., Cutrupi, M., Baronio, M., Moratto, D., Pizzino, M.R., Mankad, K., Briuglia, S., Salpietro, C. and Plebani, A. (2016) Proteus Syndrome: Evaluation of the Immunological Profile. Orphanet Journal of Rare Diseases, 11, 3. https://doi.org/10.1186/s13023-015-0381-z 